Original Article

\title{
STUDY OF ENHANCED ANTI-INFLAMMATORY POTENTIAL OF NIGELLA SATIVA IN TOPICAL NANOFORMULATION
}

\author{
FAISAL OBAID ALOTAIBI ${ }^{1}$, GULAM MUSTAFA ${ }^{1}$, ALKA AHUJA2\#
}

${ }^{1}$ College of Pharmacy (Boys), Al-Dawadmi Campus, Shaqra University, P. O. Box 11961, Riyadh, Kingdom of Saudi Arabia, ${ }^{2}$ Department of Pharmacy, Oman Medical College, P. O. Box. 620, P. C. 130, Azaiba, Muscat, Sultanate of Oman, OMAN

Email: alkaahuja@yahoo.com

Received: 06 Dec 2017 Revised and Accepted: 05 Jun 2018

\section{ABSTRACT}

Objective: Formulate a nanocarrier for enhancing the anti-inflammatory activity of thymoquinone (Tq), a major active constituent of Nigella sativa.

Methods: Nanoformulation of Tq was developed by low energy emulsification techniques. NanoTqs were pre-screened by different thermodynamic stability tests, followed by in vitro release, zeta potential, viscosity, the transmittance (\%), globule size distribution and ex vivo studies. The morphology of the optimized NanoTq was determined by transmission electron microscopy (TEM) which revealed fairly spherical shape and good correlation with particle size distribution study. The formulation used for assessment of the anti-inflammatory potential and permeability enhancement contained mixture of essential oil of Nigella sativa: Capryol $90(3: 7,10 \%$, v/v), Tween 80 (21.75\%, v/v), PEG 400 (7.25\%, v/v) and double distilled water $(61 \%, \mathrm{v} / \mathrm{v})$.

Results: The in vitro permeation of Tq from optimized formulations was found extremely significant $(\mathrm{p}<0.001)$ in comparison to apiTq. The steady state flux $(J s s)$, the permeability coefficient $(K p)$ and enhancement ratio $(E r)$ of NanoTq gel was determined and compared with apiTq. The comparative anti-inflammatory effects of the optimized formulations NanoTq, apiTq and DicloGel was assessed on the edema in the carrageenaninduced paw model in Wistar rats. Therapeutic potential of NanoTq was found statistically extremely significant $(P<0.0001)$ compared to apiTq and insignificant comparable with standard DicloGel. Storage stability of NanoTq showed insignificant changes in the zeta potential, droplet size and was free from any physical instability.

Conclusion: The optimized nano formulation with a lower dose of Tq showed better anti-inflammatory effects, indicating greater absorption capability through the stratum corneum.

Keywords: Thymoquinone, Pseudo-ternary phase diagram, Stress stability, Nanoemulsion, Anti-inflammatory, Flux

(c) 2018 The Authors. Published by Innovare Academic Sciences Pvt Ltd. This is an open access article under the CC BY license (http://creativecommons.org/licenses/by/4.0/) DOI: http://dx.doi.org/10.22159/ijpps.2018v10i7.22966

\section{INTRODUCTION}

Nigella sativa $L$ or Black seed (Family: Ranunculaceae) has been regarded as a blessing and healing food additive in the Islamic culture and extensively used as a medicinal herb [1]. Chemical analysis of Nigella sativa $L$ has shown the existence of tremendous active constituents with multifaceted therapeutic applicability. Nigella sativa Essential oil (EO) and its main active constituent, thymoquinone (Tq), are extensively reported to exhibit protective effects against many diseases largely attributable to their high antioxidant activity $[2,3]$. Thymol, thymoquinone $(\mathrm{Tq})$, thymoquinone, and carvacrol which are found in the Nigella sativa EO have been used in various therapies including as an analgesic [4], antioxidant, anti-aging [5, 6], and anticancer [7] agent and in Parkinsonism [8], Alzheimer's [9], stroke [10] and various neurological disorders. The anti-inflammatory property of these compounds have also been examined in various studies like induced rheumatoid arthritis in the rat, lung inflammation [11], eicosanoid generation in leukocytes [12] and carrageenaninduced paw edema [13]. Although Tq possesses excellent therapeutic potential but has failed to meet the clinical demand because of poor solubility and dissolution [14]. Hydrophobic drugs when delivered orally encounter permeability problems first followed by poor bioavailability and extensive hepatic metabolism. Because of these, the clinical applicability of $\mathrm{Tq}$ has been significantly hampered.

The development of transdermal drug delivery systems (TDDS) designed to have local as well as systemic effects is advisable and beneficial especially when we talk about anti-inflammatory activity. Several studies showed tremendous advancement in terms of bioavailability and acceptance that TDDS offered over conventional routes of drug administration [15-17]. But, TDDS is also restricted by the skin, a natural barrier. With the combined effort of biomedical science and engineering, chemical enhancers (synthetic and natural) and techniques such as ultrasound and iontophoresis have been designed to minimize the skin impermeability.

The use of synthetic penetration enhancers sometimes irreversibly alter the natural defence of skin and toxicity at application site [18]. Therefore, it has been advised to use novel formulation approaches to overcome permeation problem associated with penetration enhancers. A wide variety of drug carriers including nanoemulsions, microemulsions, liposomes, solid lipid nanoparticles, microspheres, and selfmicro emulsifying drug-delivery systems have been studied to improve the therapeutic efficacy of hydrophobic drugs or nutraceuticals by enhancing the bioavailability and tissue-targeting ability [17]. Among the various novel drug delivery systems, nanocarrier system has emerged as a best alternative and timely for TDDS. Nanoemulsion, a most stable nano-carrier system has combined the advantages of other innovative carrier systems with highest kinetic and thermodynamic stability, excellent acceptance and minimal inter-subject variability [19]. Nanoemulsions have been widely studied to increase the bioavailability of hydrophobic drugs [20-23]

Nanoemulsion droplet, because of its excellent flexibility can penetrate the intact stratum corneum spontaneously either through intracellular route or Trans-cellular route without much affecting the integrity of the skin [24].

Viewing the safety issue and patient acceptance, especially for local anti-inflammatory activity, the present investigation was aimed to formulate an emulsion of the nanometric size range for $\mathrm{Tq}$ to obtain local as well as systemic effects and hence avoid pre-systemic clearance. This may lead to a decrease in systemic side effects. Despite the little limiting factor, numerous advantages are associated with TDDS. It is a best alternative route for drugs with a low oral bioavailability and extensive hepatic metabolism hence 
providing controlled drug input and high patient acceptance and compliance.

\section{MATERIALS AND METHODS}

\section{Materials}

Propylene glycol monocaprylate (Capryol 90), caprylo caproyl macrogol-8-glyceride (Labrasol) and diethylene glycol monoethyl ether (Transcutol ${ }^{\circledR}$ P) were obtained from Gattefosse (Saint-Priest, Cedex, France). Propylene glycol mono caprylic ester (Safsol 218) was obtained from Nikko Chemicals (Tokyo, Japan). 2-propanol high-pressure liquid chromatography (HPLC) grade, methanol HPLC grade, and water-HPLC grade were procured from SD Fine Chemicals, Ltd. Nigella sativa EO, polyoxyethylene (20) sorbitan mono oleic acid (Polysorbate $80^{\circledR}$ ), polyoxyethylene (20) sorbitan monolaurate (Polysorbate $20^{\circledR}$ ), polyethylene glycol (PEG 200) and ethanol were purchased from GuideChem (Riyadh, KSA). Dialysis tubing (mol wt cut-off: 12,000 Da, flat with $25 \mathrm{~mm}$, diameter of 16 $\mathrm{mm}$, capacity $60 \mathrm{ml} / \mathrm{ft}$ ) was obtained from Sigma-Aldrich (St. Louis, MO, USA). All other reagents used were of analytical grade.

\section{Analytical methodology}

The content of Tq in Nigella sativa EO was estimated by reported HPLC analysis using Shimadzu LC 2010 system (Kyoto, Japan) which consisted of quaternary LC-10A VP pump, SPD-10AVP column oven and variable wavelength programmable UV/VIS detector. The analysis was carried out on a LiChrospher ${ }^{\circledR} \mathrm{C}_{18}(150 \mathrm{~mm} \times 4.6 \mathrm{~mm}$ i.d., $5 \mu \mathrm{m}$ particle size) column (Agilent Technology, California, USA). The mobile phase consisted of 2-propanol $(5 \% \mathrm{v} / \mathrm{v})$, methanol $(5 \% \mathrm{v} / \mathrm{v})$ and water $(50 \% \mathrm{v} / \mathrm{v})$ having with $\mathrm{pH} 6.8$. The flow rate was maintained at 2.0 $\mathrm{ml} / \mathrm{min}$ and the retention time was $3.2 \mathrm{~min}$. Ultraviolet detection was optimum at $294 \mathrm{~nm}$ for $\mathrm{Tq}$ [25]. The assays were performed at ambient temperature $\left(25 \pm 0.5{ }^{\circ} \mathrm{C}\right)$ and Class VP 5.03 version software was used to process the chromatograms. The same method was used for all the analytical activities.

\section{Solubility study in the oil phase and surfactant}

The solubility of Nigella sativa EO in different oil phases and surfactants was determined using isothermal water shaker maintained at $37 \pm 0.5{ }^{\circ} \mathrm{C}$. For determining the solubility in an oil phase, different ratios ( 0.01 to $0.5 \% \mathrm{v} / \mathrm{v}$ ) of oil and Nigella sativa EO were added to the stoppered vial (5 ml capacity) containing $2 \mathrm{ml}$ of an oil phase. The vials were kept in the isothermal shaker for $72 \mathrm{~h}$ for equilibration. Solubility study of Nigella sativa EO was also conducted in different co-surfactants and surfactants. The vials were visualized against the bright light to observe any phase separation or boundary layers and precipitation [26]. After $72 \mathrm{~h}$, the samples were taken out and passed through the membrane filter and the oil sample was assayed using visual clarity.

\section{Construction of pseudo-ternary phase diagram}

Pseudo-ternary phase diagrams were constructed to find out the concentration range of various components to understand the existence range in the nanophasic zone $[26,27]$. Pseudo-ternary phase diagrams of the oil phase, aqueous phase, and co-surfactant $(\mathrm{CoS}) /$ surfactants $(\mathrm{S})$ mixtures $\left(\mathrm{S}_{\text {mix }}\right)$ were constructed at fixed CoS/S weight ratios. Here, Nigella sativa EO: capryol 90 mixture was used as an internal phase (oil) whereas a mixture of tween 80 and PEG 200 was used as Smix. Distilled water was used as an external aqueous media for nanoemulsion titration. Surfactant and cosurfactant $\left(S_{\text {mix }}\right)$ were pre-mixed in different volume ratios $(1: 0,1: 1$, $1: 2,1: 3,2: 1,3: 1$, and $4: 1)$. For each phase diagram, sixteen different combinations of oil and $S_{\text {mix }}(1: 9,1: 8,1: 7,1: 6,1: 51: 4,1: 3.5,1: 3,3: 7$, $1: 2,4: 6,5: 5,6: 4,7: 3,8: 2$ and 9:1) were made. Pseudo-ternary phase diagrams were established by aqueous titration method. Slow titration with aqueous phase was done for each combination of oil and $S_{\text {mix }}$ separately [19]. The existence of nanoemulsions regions was marked on a pseudo-ternary phase diagram with axes representing the aqueous phase, oil phase and the mixture of surfactant and co-surfactant at fixed volume ratios. After constructing the pseudo-ternary phase diagrams, optimal surfactant, co-surfactant and oil combinations were selected for formulation development.

\section{Physical stress tests}

Different formulations were selected from the phase diagram showing nanoemulsion region on the basis of Nigella sativa EO miscibility with the oil phase. The minimum concentration of Smix with external phase concentration (water) must be greater than the internal phase (oil phase). To overcome the problem of metastable formulation, physical stress tests were performed $[24,26]$. To get robust formulation and to overcome the problem of metastability, physical stress tests were performed as already discussed in various studies [28].

\section{Centrifugation tests}

Selected formulations (NanoTq) were centrifuged (K241R Refrigerated Model) at $5000 \mathrm{rpm}$ for $30 \mathrm{~min}$ and observed for phase separation, creaming or cracking, turbidity and drug precipitation. The formulations that showed any of the mentioned thermodynamic stability problems were excluded from remaining stress tests.

\section{Heating cooling cycle}

Six heating-cooling cycles between heating temperature $\left(45 \pm 0.5^{\circ} \mathrm{C}\right)$ and refrigerator temperature $\left(0 \pm 0.5^{\circ} \mathrm{C}\right)$ were performed with storage at each temperature for not less than $48 \mathrm{~h}$. Those formulations, which were stable at this temperature cycle with no phase separation, creaming or cracking, turbidity and drug precipitation, were subjected to freeze and thaw cycle.

\section{Freeze-thaw cycle (Accelerated ageing)}

In freeze and thaw cycle, selected formulations were exposed to three freeze-thaw cycles between- $21 \pm 0.5{ }^{\circ} \mathrm{C}$ and $+25 \pm 0.5{ }^{\circ} \mathrm{C}$ with storage at each temperature for not less than $48 \mathrm{~h}$.

\section{In vitro permeation study}

In vitro permeation study was performed using dialysis bag supported Franz diffusion permeation cell and the mixture of acetate buffer ( $\mathrm{pH} \mathrm{5.4)}$ and Tween $20(1.0 \% \mathrm{w} / \mathrm{v})$ as a dissolution medium [29]. Only those formulations were included in permeation which survived physical and thermodynamic stress tests (table 1). In permeation study, one ml of optimized formulation (table 1) was placed in a treated dialysis bag (MWCO 12,000 g mole $^{-1}$; Sigma, St. Louis, MO, USA) and at predetermined time intervals $(0,0.25,0.5$, $0.75,1,2,5,6,10$ and $12 \mathrm{~h}) 0.5 \mathrm{ml}$ sample was withdrawn. To maintain the sink condition of dissolution medium, withdrawn volume was replenished with the same amount of fresh acetate buffer. The samples were diluted with methanol and analyzed for the drug content by reported RP-HPLC at $294 \mathrm{~nm}$ [25].

\section{Refractive index}

Refractive index $\left(R_{f}\right)$ of the optimized formulations NanoTq was determined by ABBE type refractometer. In $R_{f}$ study, cedarwood oil was used as a reference. $R_{f}$ was measured by placing drops of formulation solution on the slide.

\section{Transmittance}

Transmittance is the fraction of incident light at a specified wavelength that passes through a sample. It was assumed that, if the diameter of the dispersed phase is below the wavelength of transmitted light, it will easily pass through the sample without any absorbance. Percentage transmittance of NanoTq was measured at $630 \mathrm{~nm}$ (visible range) using UV spectrophotometer (UV 1601, Shimadzu, Japan) keeping the distilled water as a reference solution [30].

\section{Viscosity measurement}

The viscosity of the optimized formulation was estimated by Brookfield DV III ultra V6.0 RV cone and plate rheometer (Brookfield Engineering Laboratories, Inc, Middleboro, MA) with spindle \# CPE40. All measurements were carried out at a constant temperature of $25 \pm 0.5{ }^{\circ} \mathrm{C}$. The software used for the calculations was Rheocalc V2.6.

\section{Droplet size and zeta potential}

Droplet size was estimated by photon correlation spectrometer (PCS; Zetasizer-1000 HAS, Malvern Instruments, UK) which converts 
fluctuations in light scattering $\left(25^{\circ} \mathrm{C}\right.$; angle: $\left.90^{\circ}\right)$ into the diameter. Test samples were dispersed in $50 \mathrm{ml}$ (100 to $1000 \mathrm{x}$ dilutions) of distilled water and were used for droplet size analysis. Mean droplet size $(\Delta d m)$ and polydispersity index $(p i)$ were recorded.

Surface charge or zeta potential $(\zeta)$ measurements were carried out after dispersing the test samples in $50 \mathrm{ml}$ of distilled water. A potential difference across the dispersion medium was established to determine the zeta potential. The charged droplets within the dispersion medium migrated towards the electrode of opposite charge. The migration velocity is proportional to the magnitude of the zeta potential.

\section{Surface morphology (TEM)}

Surface morphology and shape of the optimized formulation were studied by transmission electron microscope (TEM) (TOPCON 002B) operating at $200 \mathrm{KV}$, capable of a point to point resolution. Diluted samples (dilution factor, 1:1000) of nanoemulsion system were used for TEM observations. The sample was negatively stained with phosphotungstic acid. A drop of diluted NanoTq was allowed to deposit directly on the circular copper film grid of 400 mesh and stained with $2 \%(\mathrm{w} / \mathrm{v})$ phosphotungstic acid for $30 \mathrm{~s}$ and placed for observation after air drying. Combination of bright field imaging at increasing magnification and diffraction modes were used to determine the form and size of the nanoemulsion.

\section{Formulation development}

The optimized nanoemulsions after in vitro characterization and optical study were converted into a gel. NanoTq Gel, apiTqGel and DicloGel was prepared after homogenous dispersion in Carbopol934 gel. Carbopol gels $(0.5 \% \mathrm{w} / \mathrm{v})$ was prepared after dispersing in distilled water and kept in dark for $24 \mathrm{~h}$ for complete swelling. The carbopol gel was stabilized by drop-wise addition of triethylamine. The optimized NanoTq, apiTq and diclofenac was added slowly into the gel with continuous stirring. The obtained gel was left for $24 \mathrm{~h}$ to get homogeneous dispersion.

\section{Ex vivo permeation study}

The rat abdominal surface hair was removed by a clipper and skin (full thickness) was surgically removed. The subcutaneous layer was removed manually and dermis side was wiped with isopropyl alcohol. The full thickness of skin was soaked in buffer solution (phosphate saline) and stored at $-20 \pm 0.5^{\circ} \mathrm{C}$ till further use. Ex vivo skin permeation was performed by the Franz diffusion permeation cell with an effective diffusion surface area of $0.636 \mathrm{~cm}^{2}$ using excised rat abdominal skin. The skin was mounted between donor and receiver compartments of the Franz diffusion cell facing stratum corneum toward the donor compartment and dermal side facing the receiver compartment. Multiple cycles, skin stabilization was done with dissolution medium. For this, the receiver chamber was filled with acetate buffer $\mathrm{pH} 5.4$ with $1 \%(\mathrm{w} / \mathrm{v})$ sodium lauryl sulfate $(S L S)$ and stirred with magnetic beads $(100 \mathrm{rpm})$ maintained at $37 \pm 0.5^{\circ} \mathrm{C}$. The whole dissolution medium was replaced with a fresh one after every $30 \mathrm{~min}$ for complete stabilization of the skin. After running 12 cycles of stabilization, $2.0 \mathrm{~g}$ of NanoTq was placed into the donor compartment. The samples were withdrawn at regular time intervals $(0.25,0.5,1,2,3,4,5,6,7,8,9,10,12,14,16$ and $24 \mathrm{~h})$. The withdrawn samples were passed through a $0.45 \mu \mathrm{m}$ membrane filter and analyzed for drug content by an RP-HPLC method.

Cumulative \% drug permeated through the skin was plotted as a function of time $(\mathrm{t})$. Permeation rate at steady state (Uss) was calculated by dividing the slope of the linear portion of graph with diffusion area. Similarly, permeability coefficient $(K p)$ was estimated by dividing Jss with an initial concentration of drug in donor cell $(D)$ :

$$
\mathrm{K}_{\mathrm{ss}} \frac{\mathrm{J}_{\mathrm{ss}}}{\mathrm{D}}
$$

The permeation enhancement ratio $(E r)$ was also calculated using formulation as given below:

$$
E_{r}=J_{s s}^{\text {Formulation }} / J_{s s}^{a p i}
$$

\section{Short term storage stability study}

The effect of short-term storage stability on droplet growth, surface charge, and drug content was estimated after storing formulations at regulated temperature and humidity for a period of $90 \mathrm{~d}$. Initially, droplet size and zeta potential measurements were carried out for NanoTq formulation. Properly sealed samples were stored at regulated temperature and humidity $\left(40 \pm 2^{\circ} \mathrm{C}, 65 \pm 5 \% \mathrm{RH}\right)$ conditions. Samples were withdrawn after $90 \mathrm{~d}$ for particle size, zeta potential and drug content measurements [24].

\section{Anti-inflammatory study}

NanoTq formulations showing excellent ex vivo permeation and short-term storage stability profile were selected for the antiinflammatory study. The anti-inflammatory studies on the optimized NanoTq formulation were performed on carrageenan-induced hind paw edema method using Wistar rats [31]. Wistar rats weighing 150-200 g, were randomized into four groups having six rats in each group. The groups were classified on the basis of formulations given like NanoTq, ApiTq, standard diclofenac and a saline control. The animals were housed in polypropylene cages ( 6 per cage), with free access to standard laboratory diet and water ad-libitum. Rat dose was calculated based on the weight of the rats according to the surface area ratio method [32]. Formulation NanoTq Gel and apiTqGel were applied on the shaved abdominal region of all animals, half an hour before sub plantar injection of salinated carrageenan suspension $(1 \% \mathrm{w} / \mathrm{v})$ in right paw. The volume of paw was measured using digital plethysmometer (LE 7500, Panlab Harvard Apparatus) at specific time intervals $(0.5,1,2,3,6$ and $12 \mathrm{~h})$. Basically, the volume transducer was formed by two Perspex tubes interconnected and filled with a conductive solution and a platinum electrode for each chamber. The water displaced after immersion of inflamed rat paw in the measuring tube was deflected into the second tube, inducing a change in the conductance between the two platinum electrodes. Per cent inhibition of edema produced by gels treated group was calculated against the respective control group and marketed DicloGel (RUMAFEN ${ }^{\circledR}$, Riyadh Pharma, Riyadh, KSA). The results of anti-inflammatory effects were compared using Dunnett test of one-way analysis of variance. The study was approved from Animal Ethical Committee, College of Pharmacy, Dawadmi Campus, Shaqra University, and Kingdom of Saudia Arabia and confirms to National guidelines on the care and use of laboratory animals.

\section{RESULTS AND DISCUSSION}

\section{Solubility study in oil phase and surfactant}

A preliminary study showed very limited nanoemulsion region when Nigella sativa EO was used as an oil phase. Therefore to get nanophasic region, dilution of Nigella sativa EO with some suitable oils was a pre-requisite. Solubility or miscibility study showed Nigella sativa EO had limited solubility in selected oil phases (fig. 1a). However, Nigella sativa EO showed good solubility in safsol 228 $(26.41 \pm 2.84 \% \mathrm{v} / \mathrm{v})$ and capryol 90 (33.34 $\pm 1.61 \% \mathrm{v} / \mathrm{v})$. Therefore capryol 90 was used as co-solvent to make the oil as an internal phase. The co-solvency study showed good miscibility of capryol 90 with Nigella sativa EO $(30 \% \mathrm{v} / \mathrm{v})$ with no phase separation even after centrifugation ( $5000 \mathrm{rpm}$ for $5 \mathrm{~min}$ ). For the present study, a combination of Nigella sativa EO (30\% v/v) and capryol $90(70 \%$ $\mathrm{v} / \mathrm{v}$ ) was selected to make the internal phase (fig. 1a).

Similarly, based on solubility data, surfactants tween 80 and PEG 200 showed significantly high $(p<0.01)$ solubility compared to cremophor EL, transcutol-P and tween 20 (fig. 1b). Therefore, tween 80 and PEG 200 were selected as a Smix for developing NanoTq formulations.

\section{Construction of pseudo-ternary phase diagram}

Pseudo-ternary phase diagrams were constructed to find out concentration range of various components for the existence range of nanophasic zone by aqueous phase titration technique at room temperature $\left(25 \pm 0.5^{\circ} \mathrm{C}\right)$. During aqueous phase titration, the metastable formulation was excluded [23]. Here polysorbate 80 and Polyethylene glycol 200 were used as a surfactant and co-surfactant respectively. Both were pre-mixed in different volume ratios to get 
maximum possible combinations. These Smix ratios were chosen in increasing concentration of co-surfactant with respect to surfactant (Surfactant: Co-surfactant: : 1:0, 1:1, 1:2, 1:3 v/v) and vice versa (Surfactant: Co-surfactant: : 2:1, 3:1, 4:1). The nanophasic area observed during phase titration was drawn the different phase diagram (fig. 2). During aqueous titration, the metastable nanogel region spontaneously converted into nanoemulsion region. Because of instability, it leads to failure of the system. Therefore, care was taken to discard formulation points coming in this region (fig. 2). In the phase diagram of Smix with high contribution of co-surfactant, a decreasing trend of the nanophasic region was observed. On the other hand, in the phase diagram of Smix with high contribution of surfactant, an increasing trend of the nanophasic area was obtained. The nanophasic area observed in Smix 1:1 to $1: 3$ was very insignificant. Similarly, the phase diagram without co-surfactant gave large liquid crystalline (LC) region or metastable region that was unable in breaking the oil-water interfacial tension. But after adding co-surfactant (Smix 4:1, 3:1 and 2:1), an increased nanophasic area was obtained. The co-surfactant made interfacial film more flexible leading to decrease in LC area. After observing nanoemulsion, it may be concluded that free energy of nanoemulsion formation is somehow dependent on the extent that how efficiently a partcular Smix lowers the interfacial tension between oil-water interface [23, 33]. The free energy and dispersion entropy lead to the spontaneous formation of thermodynamically stable nanoemulsion $[34,35]$. Therefore, while selecting the formulations composition from each phase diagrams, the care was taken to select those which could accommodate an optimum quantity of oil phase by using lowest possible Smix to further avoid the possibility of liquid crystallinity or metastability gel region.

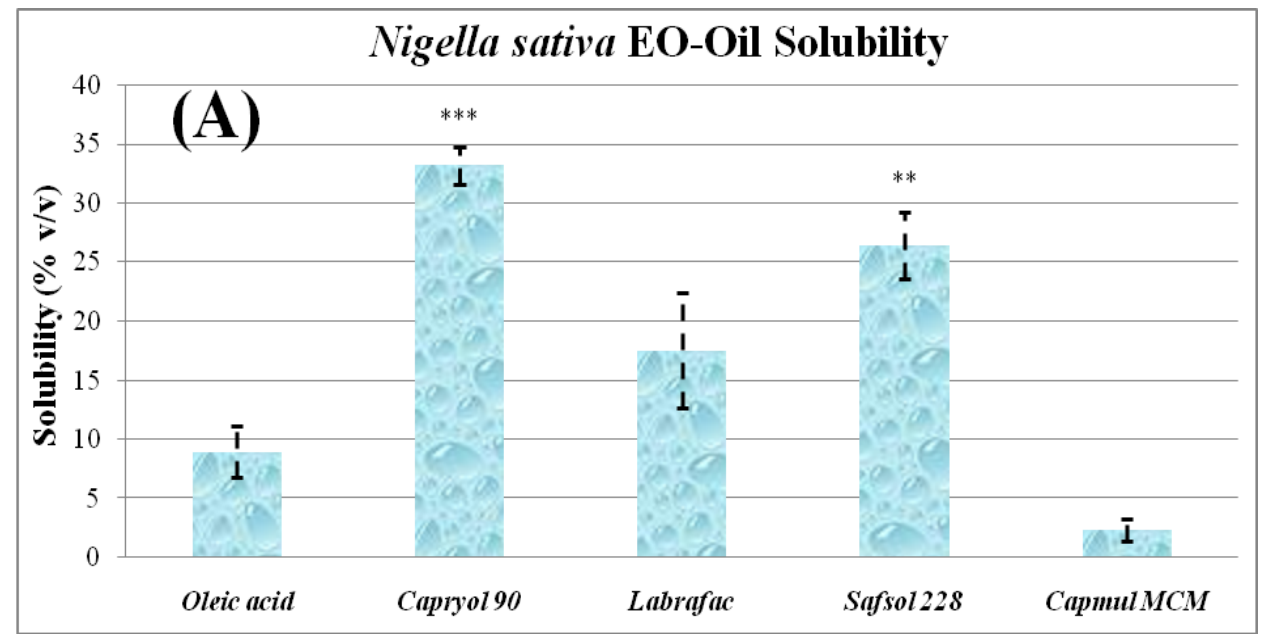

Fig. 1a: Percentage solubility study of Nigella sativa EO in different synthetic oils. The data represented as mean $\pm s d(n=3)$

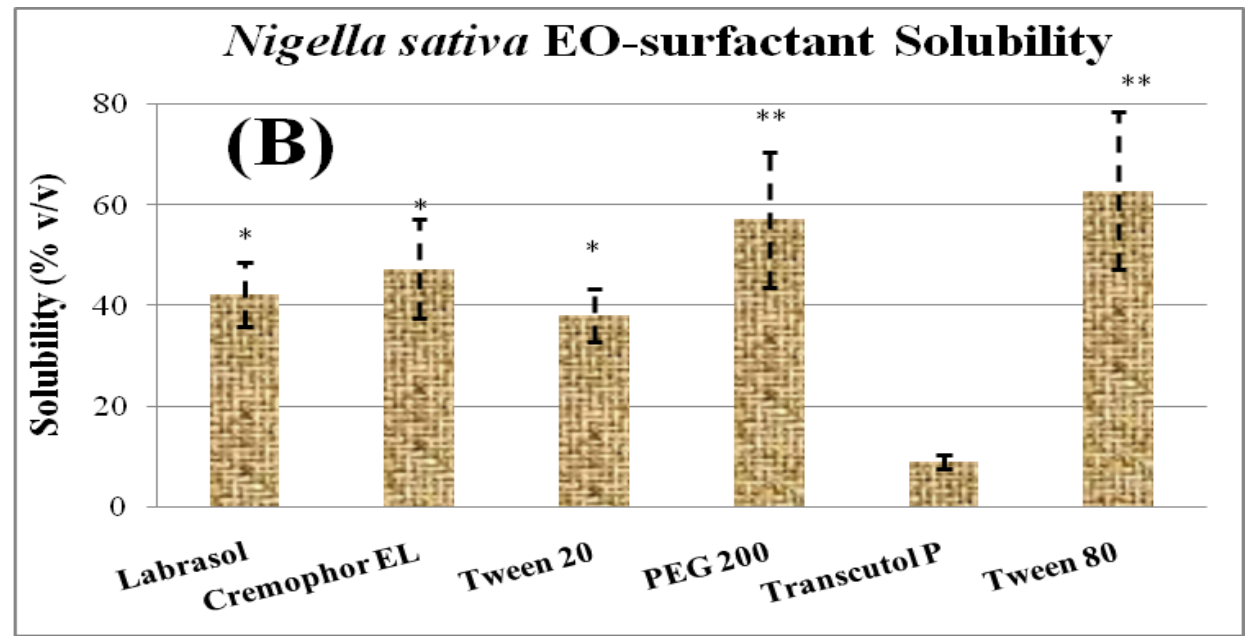

Fig. 1b: Percentage solubility study of Nigella sativa EO in different surfactants. The data represented as mean $\pm s d(n=3)$

\section{Formulation development}

The phase diagrams of Smix $1: 0$ and $1: 1$, had maximum nanophasic regions with the liquid crystalline region. The LC region, although converted spontaneously into the nanoemulsion but was unable to pass the thermodynamic stress tests. These regions comprised of the large extent of LC region whereas Smix $1: 2,1: 3$ and 1:4 gave very limited nanoemulsion region which was also associated with the LC phase in apex region. The phase diagram consisted of Smix 1:1, 2:1, 3:1 and 4:1 and showed progressive and thermodynamically stable nanoemulsion with insignificant LC and metastable regions. Different formulations were selected from different pseudo-ternary phase diagrams with justified $\mathrm{Tq}$ dose considering the drug solubility in the oils phase (table 1). Therefore, from each phase diagram different concentrations of oil, at which nanoemulsions formed, were selected at a difference of $2 \%(10,12,14,16$ and $20 \%)$ so that maximum formulations could be selected covering the nanoemulsion area of the phase diagram. Almost in all cases, Smix concentration up to $40 \%$ of the total formulation was selected. 


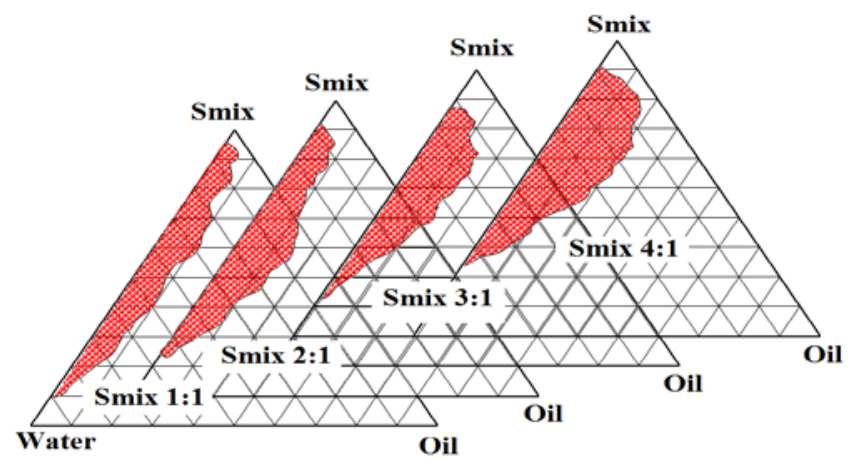

Fig. 2: Pseudo-ternary phase diagrams of nanoemulsion regions obtained using different surfactant: co-surfactant combinations (Smix)

\section{Physical stress tests}

To obtain robust formulation and to over-counter metastable, thermodynamic stability/physical stress tests (centrifugation, heatingcooling cycle and freeze-thaw cycle) are recommended [19, 27]. During stability stress testing, it was observed that some formulations became turbid and the phase separated. One reason of this instability may be due to Ostwald ripening in which stabilizing molecules move as a monomer which leads to coalescence of the smaller droplet into large droplets. The larger droplets lead to phase separation during centrifugation stress tests. Similarly, during the heating-cooling cycle, there is temperature quench leading to instability of nanoemulsion.

Table 1: Different thermodynamic stress tests of selected nano-formulations from pseudo-ternary phase diagrams

\begin{tabular}{|c|c|c|c|c|c|c|c|}
\hline \multirow{2}{*}{$\begin{array}{l}\text { Smix ratio } \\
\text { (S: CoS) }\end{array}$} & \multirow[t]{2}{*}{ Formulation code } & \multicolumn{3}{|c|}{ Compositions (v/v) } & \multicolumn{3}{|c|}{ Stress stability tests } \\
\hline & & Omix & Smix & Water & $\mathrm{H} / \mathrm{C}$ & Cent & Freez/Thaw \\
\hline $1: 0$ & $10 \%$ & 10 & 29 & 61 & $\sqrt{ }$ & $\sqrt{ }$ & $\sqrt{ }$ \\
\hline \multirow[t]{5}{*}{ (Code G) } & $12 \%$ & 12 & 31 & 57 & $\sqrt{ }$ & $\sqrt{ }$ & $\sqrt{ }$ \\
\hline & $14 \%$ & 14 & 33 & 53 & $\sqrt{ }$ & $\mathrm{x}$ & - \\
\hline & $16 \%$ & 16 & - & - & - & - & - \\
\hline & $20 \%$ & 20 & - & - & - & - & - \\
\hline & $10 \%$ & 10 & 29 & 61 & $\sqrt{ }$ & $\sqrt{ }$ & $\sqrt{ }$ \\
\hline $1: 1$ & $12 \%$ & 12 & 31 & 57 & $\sqrt{ }$ & $\sqrt{ }$ & $\sqrt{ }$ \\
\hline \multirow[t]{4}{*}{$($ Code $F)$} & $14 \%$ & 14 & 33 & 53 & $\sqrt{ }$ & $\sqrt{ }$ & $\sqrt{ }$ \\
\hline & $16 \%$ & 16 & - & - & - & - & - \\
\hline & $20 \%$ & 20 & - & - & - & - & - \\
\hline & $10 \%$ & 10 & 29 & 61 & $\sqrt{ }$ & $\sqrt{ }$ & $\sqrt{ }$ \\
\hline $1: 2$ & $12 \%$ & 12 & 31 & 57 & $\sqrt{ }$ & $\sqrt{ }$ & $\sqrt{ }$ \\
\hline \multirow[t]{4}{*}{ (Code E) } & $14 \%$ & 14 & 33 & 53 & $\sqrt{ }$ & $\sqrt{ }$ & $\sqrt{ }$ \\
\hline & $16 \%$ & 16 & - & - & - & - & - \\
\hline & $20 \%$ & 20 & - & - & - & - & - \\
\hline & $10 \%$ & 10 & 29 & 61 & $\sqrt{ }$ & $\sqrt{ }$ & $\sqrt{ }$ \\
\hline $1: 3$ & $12 \%$ & 12 & 31 & 57 & $\sqrt{ }$ & $\sqrt{ }$ & $\sqrt{ }$ \\
\hline \multirow[t]{4}{*}{ (Code D) } & $14 \%$ & 14 & 33 & 53 & $\sqrt{ }$ & $\mathrm{x}$ & - \\
\hline & $16 \%$ & 16 & - & - & - & - & - \\
\hline & $20 \%$ & 20 & - & - & - & - & - \\
\hline & $10 \%$ & 10 & 29 & 61 & $\sqrt{ }$ & $\sqrt{ }$ & $\sqrt{ }$ \\
\hline $2: 1$ & $12 \%$ & 12 & 31 & 57 & $\sqrt{ }$ & $\sqrt{ }$ & $\sqrt{ }$ \\
\hline \multirow[t]{4}{*}{$($ Code $C)$} & $14 \%$ & 14 & 33 & 53 & $\sqrt{ }$ & $\sqrt{ }$ & $\sqrt{ }$ \\
\hline & $16 \%$ & 16 & 35 & 49 & $\sqrt{ }$ & $\sqrt{ }$ & $X$ \\
\hline & $20 \%$ & 20 & - & - & - & - & - \\
\hline & $10 \%$ & 10 & 29 & 61 & $\sqrt{ }$ & $\sqrt{ }$ & $\sqrt{ }$ \\
\hline $3: 1$ & $12 \%$ & 12 & 31 & 57 & $\sqrt{ }$ & $\sqrt{ }$ & $\sqrt{ }$ \\
\hline \multirow[t]{4}{*}{ (Code B) } & $14 \%$ & 14 & 33 & 53 & $\sqrt{ }$ & $\sqrt{ }$ & $\sqrt{ }$ \\
\hline & $16 \%$ & 16 & 35 & 49 & $\mathrm{x}$ & - & - \\
\hline & $20 \%$ & 20 & 37 & 43 & $\mathrm{x}$ & - & - \\
\hline & $10 \%$ & 10 & 29 & 61 & $\sqrt{ }$ & $\sqrt{ }$ & $\sqrt{ }$ \\
\hline $4: 1$ & $12 \%$ & 12 & 31 & 57 & $\sqrt{ }$ & $\sqrt{ }$ & $\sqrt{ }$ \\
\hline \multirow[t]{3}{*}{ (Code A) } & $14 \%$ & 14 & 33 & 53 & $\sqrt{ }$ & $\sqrt{ }$ & $\sqrt{ }$ \\
\hline & $16 \%$ & 16 & 35 & 49 & $\sqrt{ }$ & $\mathrm{x}$ & - \\
\hline & $20 \%$ & 20 & 37 & 43 & $\sqrt{ }$ & $\mathrm{x}$ & - \\
\hline
\end{tabular}

Note: Tq-PEG (1:1) mixture (Omix), Surfactant-Co-surfactant ration (Smix), Heating-cooling cycle (H/C), Centrifugation study (Cent), Freeze-thaw cycle (Freez-Thaw).

The temperature quenching lead to small droplet size which favours Brownian movement which helps in efficiently re-distributing the particles to an equilibrium configuration with the lowest total free energy [35].
Sometimes, over dilution leads to leaching the stabilizers from interface to the bulk medium. This process also hastens during centrifugation stress and hence phases separation. Formulations that showed no phase separation, creaming, cracking, coalescence 
and phase inversion during stress stability tests were selected (table 1).

\section{In vitro permeation study}

The release of Tq from optimized formulations consisting of $10 \%$ oil phase was significantly high $(p<0.01)$ compared to formulations consisting of $12 \%$ oil phase. Drug release from NanoTq B was very rapid initially, as $91 \%$ of the drug was released in $12 \mathrm{~h}$. When we compared $\geq 80 \%$ drug release graph (fig. 3 ), it was very clear that most of the formulations with $10 \%$ oil composition released approx $80 \%$ drug content in less than $8 \mathrm{~h}$. Compared to $10 \%$ composition, only two formulations i.e. NanoTq B and NanoTq C consisting of $12 \%$ oil phase were able to release $80 \%$ drug in quick fashion. This could be the consequences of efficient droplet nanosizing and least polydispersity index. The order of drug release pattern from optimized formulations with $12 \%$ oil phase were NanoTq B $\left(S_{\text {mix }}\right.$ 3:1)>NanoTq C $\left(S_{\text {mix }} 2: 1\right)>$ NanoTq F $\left(S_{\text {mix }} 1: 1\right)>$ NanoTq A $\left(S_{\text {mix }}\right.$ $4: 1)>$ NanoTq E $\left(S_{\text {mix }} 1: 2\right)>$ NanoTq D $\left(S_{\text {mix }} 1: 3\right)$ in $12 \mathrm{~h}$. While, drug release order from formulations with $10 \%$ oil phase were NanoTq B $\left(\mathrm{S}_{\text {mix }} 3: 1\right)>\operatorname{NanoTq} C\left(\mathrm{~S}_{\text {mix }} 2: 1\right)>\operatorname{NanoTq} A\left(\mathrm{~S}_{\text {mix }} 4: 1\right)>\operatorname{NanoTq} \mathrm{F}\left(\mathrm{S}_{\text {mix }}\right.$ 1:1)>NanoTq E $\quad\left(S_{\text {mix }}\right.$ 1:2)>NanoTq $\quad D \quad\left(S_{\text {mix }}\right.$ 1:3). Although formulations consisting of $10 \%$ oil phase were rapid in drug release but they were found to be metastable nanogels. It was found that metastability interfered with emulsification leading to varied drug release pattern (fig. 3).

\section{Transmittance and refractive index}

The droplet size of formulations is inversely proportional to percentage transmittance; therefore higher the value of $\% \mathrm{~T}$, lower the droplet size of the formulation [28].

The percentage transmittance is based on UV-spectrophotometer which uses visible light wavelength. The \%T approaching 100\% indicates that the dispersed phase and dispersion medium are alike which means isotropy with aqueous phase. The results showed that $\%$ $\mathrm{T}$ of all selected NanoTq formulation ranged between $91.34 \pm 21.35$ to $98.67 \pm 7.13$ (table 2).
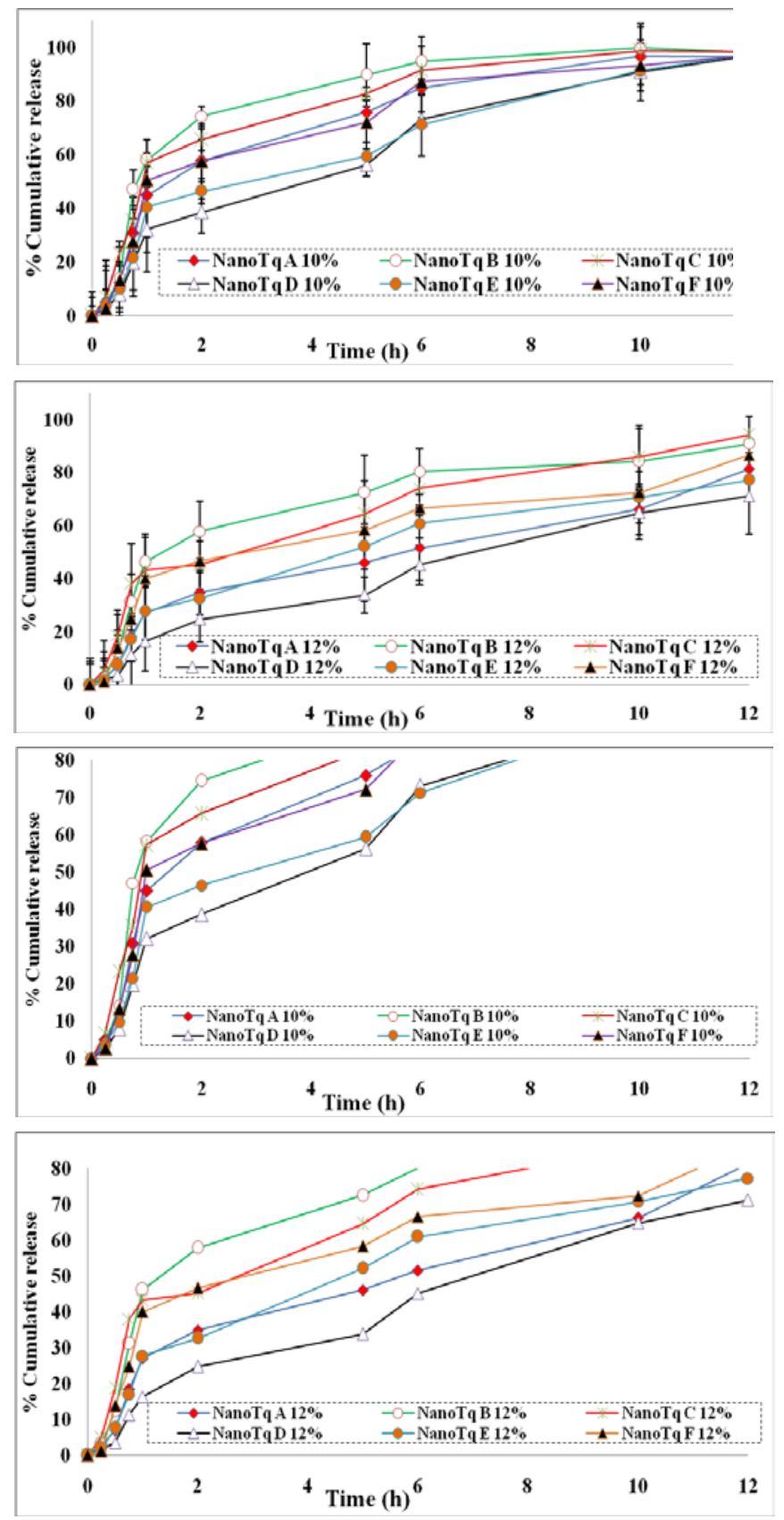

Fig. 3: Permeation study of optimized formulation. The data presented as the mean $\pm s d(n=3)$ 
Table 2: Comparative characterization of the optimized formulations

\begin{tabular}{|c|c|c|c|c|c|}
\hline Formulation & $\begin{array}{l}\text { Transmittance (\%T) } \\
\text { (mean } \pm \text { sd) }\end{array}$ & $\begin{array}{l}\text { Refractive index }\left(R_{f}\right) \\
(\text { mean } \pm s d)\end{array}$ & $\begin{array}{l}\text { Droplet size (nm) } \\
\text { (mean } \pm \text { sd) }\end{array}$ & $\begin{array}{l}\text { Viscosity (mPas) } \\
\text { (mean } \pm \text { sd) }\end{array}$ & $\begin{array}{l}\text { Zita potential }(\mathrm{mV}) \\
(\mathrm{mean} \pm \mathrm{sd})\end{array}$ \\
\hline NanoTq A10\% & $96.12 \pm 15.56$ & $1.369 \pm 0.0239$ & $107.28 \pm 19.43$ & $142.09 \pm 23.04$ & $32.43 \pm 4.19$ \\
\hline NanoTq B10\% & $98.67 \pm 7.13$ & $1.359 \pm 0.0205$ & $87.13 \pm 11.33$ & $134.74 \pm 26.17$ & $31.53 \pm 5.38$ \\
\hline NanoTq C10\% & $101.71 \pm 11.92$ & $1.362 \pm 0.0273$ & $96.44 \pm 13.64$ & $128.49 \pm 15.82$ & $27.03 \pm 2.53$ \\
\hline NanoTq D10\% & $94.34 \pm 21.35$ & $1.364 \pm 0.0264$ & $122.93 \pm 15.16$ & $103.91 \pm 11.52$ & $20.47 \pm 7.39$ \\
\hline NanoTq E10\% & $95.55 \pm 18.42$ & $1.365 \pm 0.0216$ & $112.17 \pm 17.35$ & $109.12 \pm 15.26$ & $22.01 \pm 5.34$ \\
\hline NanoTq F10\% & $96.43 \pm 10.26$ & $1.366 \pm 0.0259$ & $102.95 \pm 21.42$ & $118.15 \pm 13.88$ & $26.21 \pm 6.63$ \\
\hline
\end{tabular}

Note: All data is the average finding of three experimental outcome and expressed as the mean \pm standard deviation. The notation used are; transmittance $(\% \mathrm{~T})$, refractive index $\left(\mathrm{R}_{\mathrm{f}}\right)$, particle size distribution $(\Sigma \mathrm{d})$, viscosity $\left(\eta_{\mathrm{m}}\right)$, zeta potential $(\zeta)$, polydispersity index $($ pi), standard deviation (sd), milli volt (mV).

The decreasing order of percentage transmittances were as NanoTq C10\%>NanoTq $\quad$ B10\%>NanoTq $\quad \mathrm{F} 10 \%>$ NanoTq $\quad$ A10\% $\%$ NanoTq E10\%>NanoTq D10\%. The optimized formulation NanoTq C10\% obtained from Smix 2:1 phase diagram showed maximum transmittance. Formulations consisting of high Smix 4:1 to 2:1 ratio showed the increasing order of \%T. On the other hand, formulations consisting of Smix 1:3 to 1:1 ratio showed the increasing order of $\% \mathrm{~T}$. The results conclude that nanosizing and transmittance are the combined effects of Smix on interfacial film flexibility.

Refractive index $\left(\mathrm{R}_{\mathrm{f}}\right)$ is used to characterize the isotropic nature of the nanoformulations and also suggests the colour chemical interaction, turbidity and precipitation among drug and excipients. The $R_{f}$ value of all the optimized formulations are mentioned in the table (table 2). The least value of $\mathrm{R}_{\mathrm{f}}$ was $1.359 \pm 0.0205$ for NanoTq $\mathrm{B} 10 \%$ whereas it was maximum for NanoTq A10\% respectively.

\section{Viscosity measurement}

The viscosity of the optimized formulations was determined as shown in table 2 . The viscosity results can be correlated with the different ratios of the surfactants as well as co-surfactants used in the stabilization of selected formulation composition. It was observed that the viscosity of all the formulations was less than 145 mPas. Formulation viscosity of NanoTq D and NanoTq E was shown as $103.91 \pm 11.52$ and $109.12 \pm 15.26$ mPas respectively which was significantly $(p<0.01)$ low as compared to the other formulations (table 2). The low viscosities of all formulations met the expected criteria as required for better emulsification and spreadability.

\section{Droplet size and zeta potential}

The droplet size distribution and surface charge analysis were performed by Malvern zetasizer (Nano ZS-90 UK). The mean droplet sizes of the differently optimized nanoemulsions are shown in table 2. It was observed that in all cases diameters of the optimized formulations were below $125 \mathrm{~nm}$, proving the existence of nanoemulsion region (fig. 4A). The order of average droplet sizes were NanoTq D10\% $(122.93 \pm 15.16 \quad \mathrm{~nm})>$ NanoTq $\quad$ E10\%
$(112.17 \pm 17.35 \mathrm{~nm})>$ NanoTq $A 10 \%(107.28 \pm 19.43 \mathrm{~nm})>$ NanoTq F10\% $\quad(102.95 \pm 21.42 \quad \mathrm{~nm})>\mathrm{NanoTq} \quad \mathrm{C} 10 \% \quad(96.44 \pm 13.64$ $\mathrm{nm})>$ NanoTq B10\% $(87.13 \pm 11.33 \mathrm{~nm})$. The droplet size results showed that compositional variability is the major deciding factor for interfacial film flexibility and hence droplet size. The flexibility of interfacial films is a pre-requisite for effective nanosizing [36]. Results showed that co-surfactant aided in creating sufficient flexibility to the interfacial films that is required for secondary nanosizing, but it will not be effective for nanosizing if Smix is below the required HLB. Similarly, high surfactant concentration although increases the HLB value but the droplet size was more, which may be the consequence of rigid interfacial film leading to improper emulsification and hence nanosizing. Therefore, proper composition selection and functional role of different components is important for nanosizing and hence optimum bioavailability. Similar to flexible interfacial films, zeta potential or surface charge is equally important in deciding the fate of formulation stability. The zeta potential close to $\pm 25 \mathrm{mV}$ minimizes the contact angle and hence provides maximum stability [24]. The zeta potential results of all the optimized formulations varied between $20.47 \pm 7.39$ to $31.53 \pm 5.38$ $\mathrm{mV}$ (table 2) which comes under safe stability range (fig. 4B). The order of zeta potential were found to be NanoTq D10\% (20.47 \pm 7.39 $\mathrm{mV})>$ NanoTq $\mathrm{E} 10 \%(22.01 \pm 5.34 \mathrm{mV})>\mathrm{NanoTq} \mathrm{A} 10 \%(32.43 \pm 4.19$ $\mathrm{mV})>$ NanoTq F10\% $(26.21 \pm 6.63 \mathrm{mV})>$ NanoTq $\mathrm{C} 10 \%(27.03 \pm 2.53$ $\mathrm{mV})>$ NanoTq B10\% $(31.53 \pm 5.38 \mathrm{mV})$.

\section{Surface morphology (TEM)}

Transmission electron microscopy is the most important technique to study the shape of nanometric structures because it directly produces the point to point images at high resolution. The morphology of droplet determined by TEM is shown in fig. 4. The morphology of Tq loaded nanodroplet (nearly spherical), could be observed in the fig. TEM imaging of the optimized NanoTq has shown a good agreement with the droplet size distribution data (fig. 5, table 2). The droplet had smooth edges and was uniformly distributed. Overall, the results justifying the narrow polydispersity index was as obtained in the particle size distribution study.

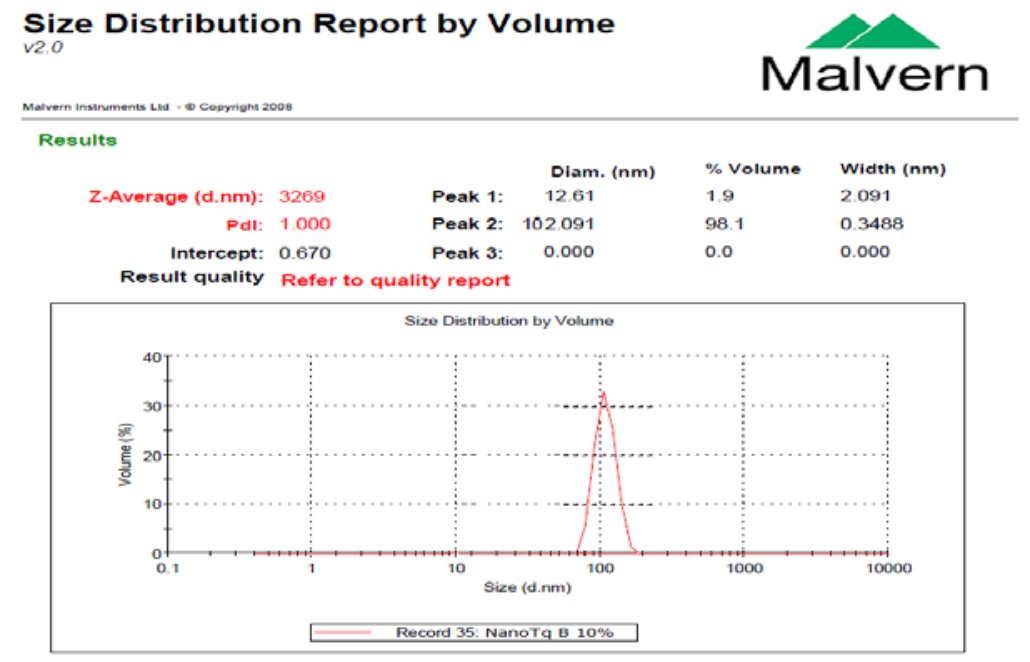




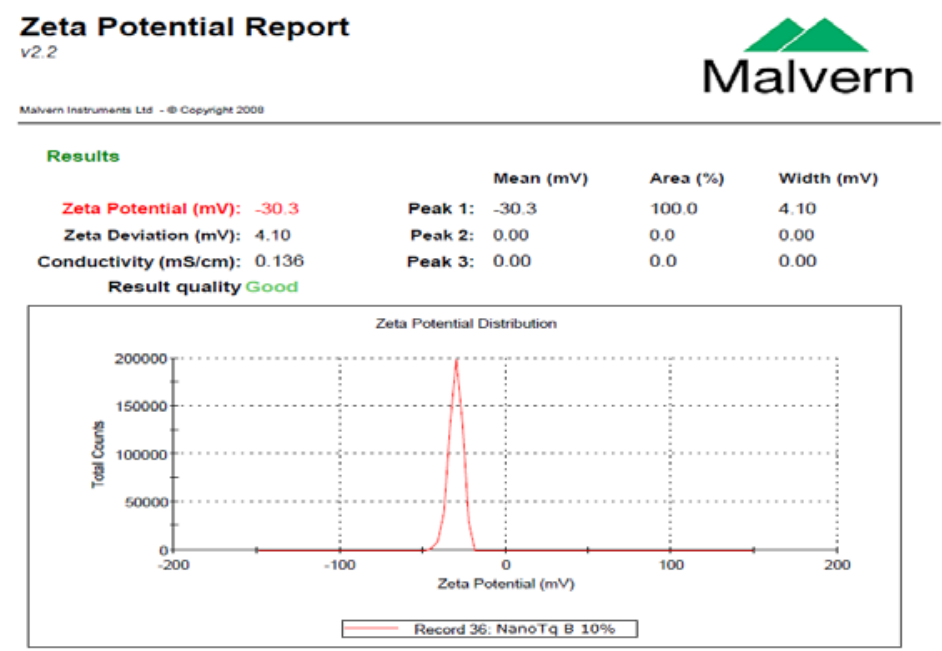

Fig. 4: Dynamic light scattering technique for determining the particle size distribution (A) and zeta potential (B) of the optimized formulation (NanoTq B 10\%)

\section{Ex vivo permeation study}

Ex vivo skin permeation studies were done to compare permeation profile of Tq from different NanoTq formulations and suspension of apiTq (ApiTqGel) using a small fragment of abdominal rat skin as a permeation barrier (fig. 6). Acetate buffer ( $\mathrm{pH} 5.4)$ with $1 \%(\mathrm{w} / \mathrm{v})$ sodium lauryl sulfate $(S L S)$ was added in receiver compartment of Franz diffusion cell in order to maintain the sink condition. Maximum ex vivo skin permeation was exhibited for NanoTq $10 \%$ and lowest in case of ApiTqGel (fig. 5). Ex vivo permeation showed good correlation with dialysis bag supported in in vitro permeation study. The small droplet size with the larger surface area, least polydispersity index, and favorable zeta potential is the favorable condition for enhancing the flux of NanoTq $10 \%$ [37]. The lipidic bilayer nature of stratum corneum favours easy dissolution of the drug encapsulating in lipid domain $[18,26]$. Similarly, water is a natural penetration enhancer for both hydrophilic and hydrophobic molecules. Hydrated skin, causing reversible separation of lipid bilayers of SC intercellular space to form cisternae, and networks of spherical particulates are observed in porcine skin tissue after 4-10 h treatment with water $[18,38]$. Furthermore, PEG 200 which is present in the optimized formulation causes an alteration in the stratum corneum and thereby aids in partitioning for better absorption.

Permeability flux (Uss), permeability coefficient $(K p)$ and permeation enhancement ratio (Er) were significantly increased by NanoTq $10 \%$ compared to ApiTqGel $(p<0.001)$. The particle size difference of NanoTq A10\% and NanoTq F10\% formulations was insignificant but the flux difference was quite significant. The result showed the significance of compositional variable which not only decides the droplet size but also the flexibility of interface required for renanosizing and permeation. The order of steady-state flux calculated for the selected formulations was NanoTq $\mathrm{A} 10 \%>\mathrm{NanoTq}$ B10\%>NanoTq $\quad$ C10\%>NanoTq $\quad$ D10\%>NanoTq $\quad$ E10\%>NanoTq F10\%, as reported in table 3. Surprisingly, the flux of NanoTq B and NanoTq C were found almost similar, although there were different particle size distribution and Smix ratio. This proved the significance of interfacial film in permeation. The comparative permeation enhancement ratio was highest for NanoTq $\mathrm{A} 10 \%$ and least for NanoTq F10\%. Other permeability parameters of different formulations are shown in table 3 .

Table 3: Comparative Ex vivo permeability parameter of different NanoTq formulation and NeatTq formulation

\begin{tabular}{|c|c|c|c|}
\hline Formulation & Flux $\left(J s s \pm s d ; \mu g / \mathrm{cm}^{2} / \mathrm{h}\right)$ & Permeability constant $(K p \pm s d ; c m / h)$ & Enhancement ratio $(E r)$ \\
\hline NanoTq A10\% & $6.354 \pm 0.635^{* *}$ & $0.116 \pm 0.075$ & $4.37^{* *}$ \\
\hline NanoTq B10\% & $5.701 \pm 0.865^{* *}$ & $0.104 \pm 0.06$ & $3.92^{* *}$ \\
\hline NanoTq C10\% & $5.691 \pm 0.792^{* *}$ & $0.103 \pm 0.012$ & $3.91^{* *}$ \\
\hline NanoTq D10\% & $5.424 \pm 0.537^{*}$ & $0.099 \pm 0.056$ & $3.73^{*}$ \\
\hline NanoTq E10\% & $5.415 \pm 0.362 *$ & $0.096 \pm 0.017$ & $3.72^{*}$ \\
\hline NanoTq F10\% & $5.158 \pm 0.819^{* *}$ & $0.106 \pm 0.014$ & $3.55^{* *}$ \\
\hline NeatTq & $1.452 \pm 0.635$ & $0.026 \pm 0.019$ & - \\
\hline
\end{tabular}

Note: Data presented here is the average of three experiments trial and expressed as the mean \pm standard deviation. Here $\mathrm{p}^{*}<0.05$, $\mathrm{p}^{*}<0.01$ and $\mathrm{p}^{*}<0.001$

\section{Short term storage stability studies}

Short term storage stability of the optimized formulations was evaluated by size distribution and zeta potential measurements using dynamic light scattering; visual observation of macroscopic changes including phase separation or creaming and drug precipitation. After storing for $180 \mathrm{~d}\left(45 \pm 0.5^{\circ} \mathrm{C} / 75 \% \pm 0.5 \mathrm{RH}\right)$, the formulations were critically evaluated. Visual observation showed no phase separation and drug precipitation. Similarly droplet size study and zeta potential showed very insignificantly $\left(\mathrm{p}^{*}<0.001\right)$ changes as shown in table 4 .

\section{Anti-inflammatory study}

Comparative anti-inflammatory effects of the optimized nanoformulation (NanoTq) and apiTq were evaluated using
Plethysmometer. The water displaced after immersion of rat paw in measuring tube changed the conductance between platinum electrodes. The percentage anti-inflammatory effects post $12 \mathrm{~h}$ carrageenan application followed by NanoTq application was insignificant for NanoTq B $10 \%(72.54 \%)$ as compared to standard DicloGel $(79.26 \%)$ were as highly significant $\left(p^{*}<0.001\right)$ as compared to apiTqGel $(26.86 \%)$ as shown in fig. 7.

The effectiveness of apiTqGel was very poor even after $12 \mathrm{~h}$. This may be because of poor solubility and dissolution problem leading to poor permeation (fig. 6 and 7).

Earlier report favours significant suppression of monocyte chemo attractant protein-1 promoter, inhibition of TNF- $\alpha$, IL-1 $\beta$, NO, PGE2, production of NF-kappa B, and reduced transport of NF-kappa B 
from the cytosol to the nucleus by $\mathrm{Tq}[39,40]$. The enhanced antiinflammatory effects of NanoTq were due to efficient penetration and attainment of high therapeutic concentration at the localized area as well as into the systemic circulation. The macromolecular size of aqueous $\mathrm{Tq}$ solution compared to a nanometric range of NanoTq caused inefficient penetration.

Table 4: Storage stability study performance at a regulated temperature $\left(45 \pm 0.5^{\circ} \mathrm{C}\right)$ and humidity $(75 \% \pm 0.5 \mathrm{RH})$

\begin{tabular}{|c|c|c|c|c|c|c|}
\hline \multirow[t]{2}{*}{ Formulation } & \multicolumn{2}{|c|}{ Day one sampling } & \multicolumn{4}{|c|}{ Day $90^{\text {th }}$ sampling $\left(45 \pm 0.5^{\circ} \mathrm{C} / 75 \% \pm 0.5 \mathrm{RH}\right)$} \\
\hline & $\Sigma \mathrm{d} \pm \mathrm{sd}(\mathrm{nm})$ & $\zeta \pm s d(\mathrm{mV})$ & $\Sigma d \pm s d(n m)$ & $\Delta d(\%)$ & $\zeta \pm s d(\mathrm{mV})$ & $\Delta \zeta(\%)$ \\
\hline NanoTq A10\% & $107.28 \pm 19.43$ & $36.43 \pm 4.19$ & $113.26 \pm 9.35$ & 5.57 & $31.92 \pm 3.68$ & 12.38 \\
\hline NanoTq B10\% & $87.13 \pm 11.33$ & $31.53 \pm 5.38$ & $89.62 \pm 8.47$ & $2.86^{* *}$ & $30.23 \pm 1.18$ & $4.12^{* *}$ \\
\hline NanoTq C $10 \%$ & $96.44 \pm 13.64$ & $28.03 \pm 2.53$ & $101.33 \pm 7.91$ & 5.07 & $26.63 \pm 4.03$ & $4.99 * *$ \\
\hline NanoTq D10\% & $122.93 \pm 15.16$ & $24.47 \pm 7.39$ & $128.85 \pm 10.45$ & 4.82 & $19.19 \pm 3.47$ & 21.58 \\
\hline NanoTq E10\% & $112.17 \pm 17.35$ & $25.01 \pm 5.34$ & $119.40 \pm 9.93$ & 6.45 & $22.41 \pm 4.12$ & 10.40 \\
\hline NanoTq F10\% & $102.95 \pm 21.42$ & $26.21 \pm 6.63$ & $111.38 \pm 8.12$ & 8.19 & $22.89 \pm 4.97$ & 12.67 \\
\hline
\end{tabular}

Note: Particle size distribution $(\Sigma \mathrm{d})$, Zeta potential $(\zeta)$, Standard deviation (sd), milli volt $(\mathrm{mV})$, Experimental data were triplicate and expressed as the mean \pm standard deviation $(\mathrm{n}=3)$

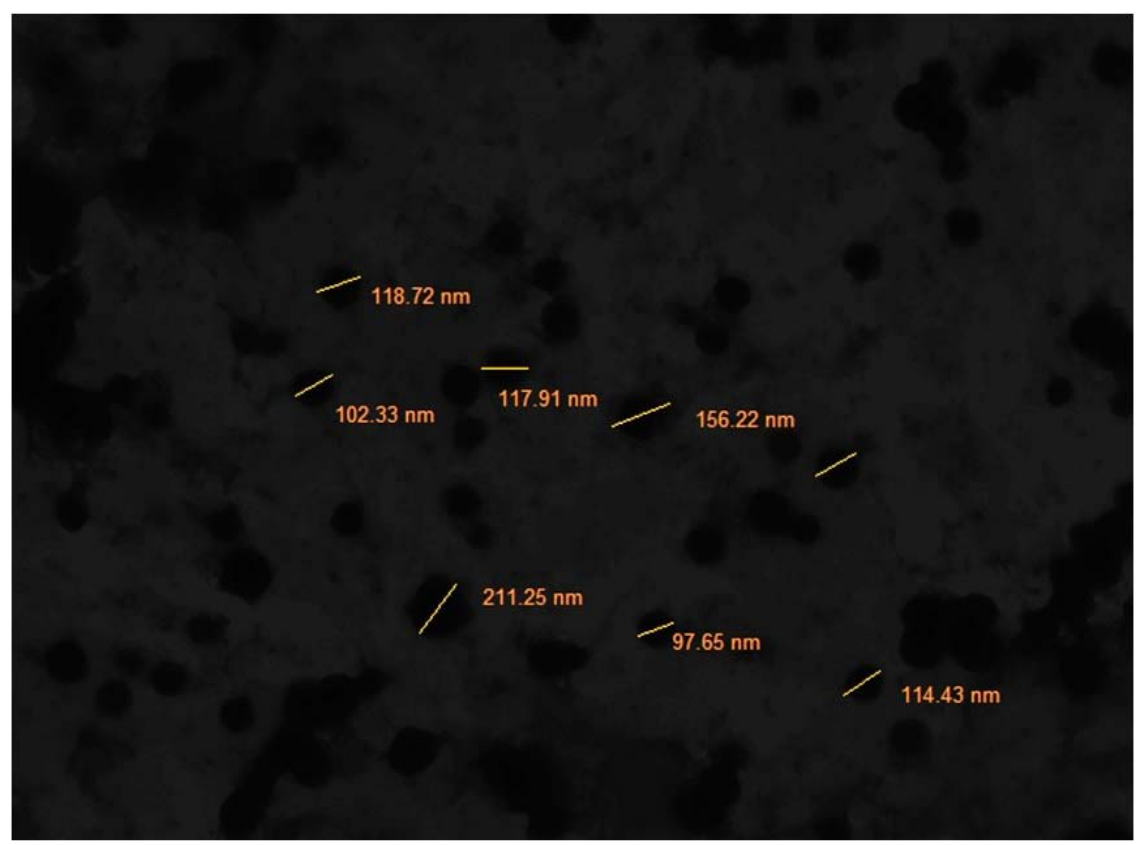

Fig. 5: Transmission electron microscopic (TEM) imaging of NanoTq showing the shape of oil-droplet

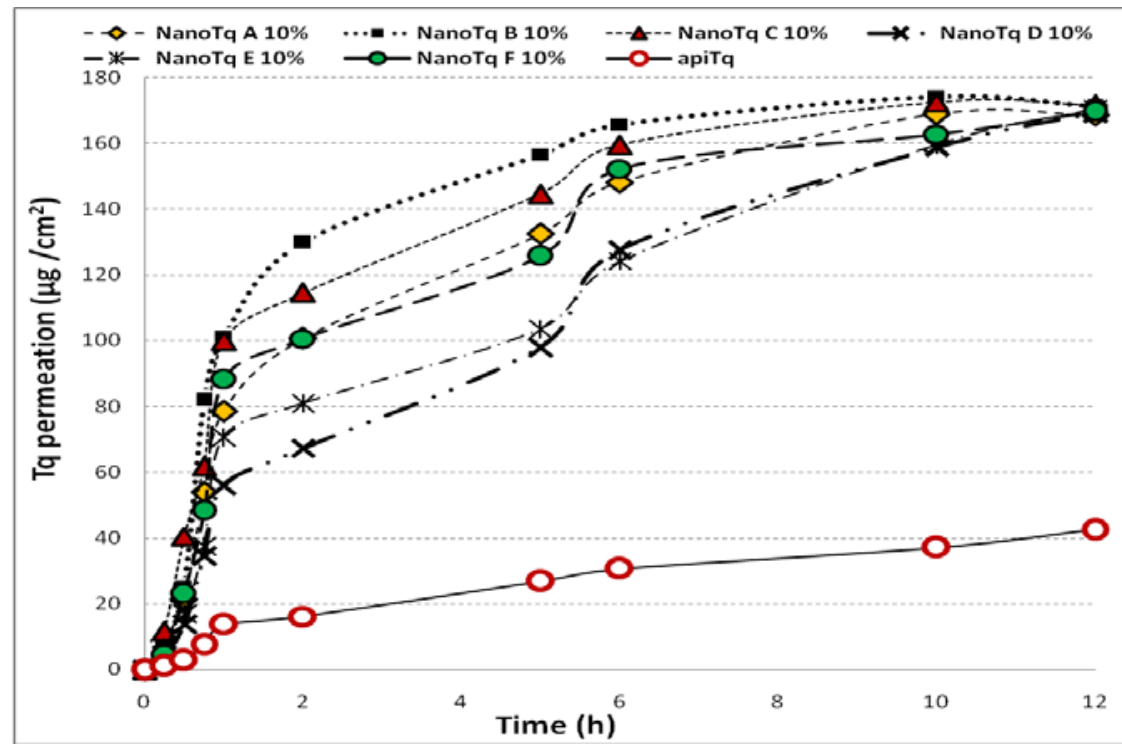

Fig. 6: Comparative ex vivo permeation study of optimized Tq formulations (NanoTq A-F) and apiTq using Franz diffusion cell. Acetate buffer (pH 5.4) with PEG 200 (1\% w/v) was used as dissolution medium 


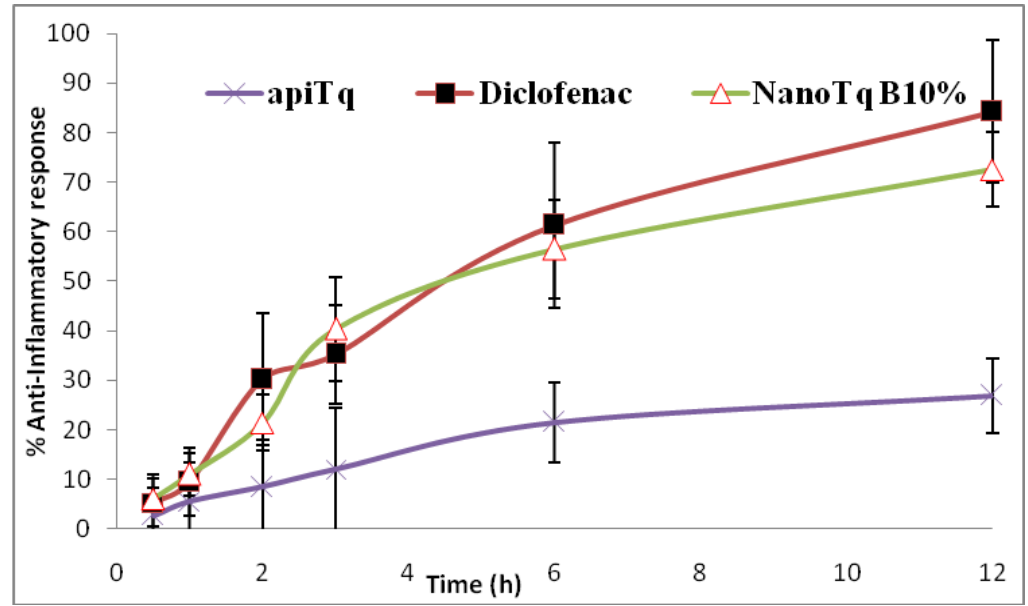

Fig. 7: Comparative anti-inflammatory response of (a) standard diclofenac suspension (b) NanoTq and (c) apiTq on the carragenaninduced paw edema in rats. Each point represents mean \pm sd $(\mathrm{n}=6)$. Here $p^{*}<0.005, p^{* *}<0.001$ and $p^{* * *}<0.0001$

\section{CONCLUSION}

The composition of nanoparticulate formulations is playing an important role in the effective transdermal delivery system. On the basis of polydispersity index, zeta potential, droplet size, drug permeation study and optimum Smix concentration, NanoTq B 10\% was selected as an optimized formulation. Anti-inflammatory activity of the optimized NanoTq was increased with the shortened lag times in comparison to apiTq. Short term storage stability at regulated temperatures and humidity showed insignificant changes in mean diameters, zeta potential with no any observable physical changes. This proved good shelf-life of the optimized formulation. The carrageenan-induced rat paw edema results further potentiate NanoTq B $10 \%$ as a promising vehicle for improved transdermal delivery. Anti-inflammatory potential of NanoTq B was found equally effective as standard DicloGel whereas significantly high compared to apiTq. Thus, the optimized formulation NanoTq B could be used as an effective potential anti-inflammatory agent. These studies no doubt need further optimization to make the optimized formulation more effective.

\section{ACKNOWLEDGEMENT}

The authors are thankful to College of Pharmacy, Shaqra University, Riyadh, KSA for providing the facility. The Authors are also thankful to Gattefosse (Saint-Priest, Cedex France) and Nikko chemical Ltd (Japan) for Transcutol and sefsol 218 respectively. Authors are thankful to Grammarly which helped in correcting the manuscript.

\section{AUTHORS CONTRIBUTIONS}

Authors have no any conflict of interest

\section{CONFLICT OF INTERESTS}

Declared none

\section{REFERENCES}

1. Sharrif MM. Nigella sativa traditional usages (Black Seed). Adv Environ Biol 2011;5:5-16.

2. AL-Naqeeb G, Ismail M. Regulation of apolipoprotein A-1 and apolipoprotein B100 genes by thymoquinone rich fraction and thymoquinone in hepg2 cells. J Food Lipids 2009;16:245-58.

3. Mariod AA, Ibrahim RM, Ismail M, Ismail N. Antioxidant activity and phenolic content of phenolic-rich fractions obtained from black cumin (nigella sativa) seedcake. Food Chem 2009;116:306-12.

4. Houghton PJ, Zarka R, de Las Heras B, Hoult JR. Fixed oil of Nigella sativa and derived thymoquinone inhibit eicosanoid generation in leukocytes and membrane lipid peroxidation. Planta Med 1995;61:33-6.

5. Nagi MN, Alam K, Badary OA, Al-Shabanah OA, Al-Sawaf HA, AlBekairi AM. Thymoquinone protects against carbon tetracholide hepatotoxicity in mice via an antioxidant mechanism. Biochem Mol Biol Int 1999;47:153-9.
6. Burits M, Bucar F. Antioxidant activity of nigella sativa essential oil. Phytother Res 2000;14:323-8.

7. Kumara SS, Huat BT. Extraction, isolation and characterization of anti-tumour principle, alpha-hedrin, from the seeds of nigella sativa. Planta Med 2001;67:29-32.

8. Sedaghat R, Roghani M, Khalili M. Neuroprotective effect of thymoquinone, the nigella sativa bioactive compound, in a 6hydroxydopamine-induced hemi-parkinsonian rat model. Iran J Pharm Res 2014;13:227-34.

9. Ismail N, Ismail M, Mazlan M, Latiff LA, Imam MU, Iqbal S, et al. Thymoquinone prevents $\beta$-amyloid neurotoxicity in primary cultured cerebellar granule neurons. Cell Mol Neurobiol 2013;33:1159-69.

10. Akhtar M, Maikiyo AM, Najmi AK, Khanam R, Mujeeb M, Aqil M. Neuroprotective effects of chloroform and petroleum ether extracts of nigella sativa seeds in stroke model of rat. J Pharm Bioallied Sci 2013;5:119-25.

11. El-Gazzar M, El-Mezayen R, Marecki JC, Nicolls MR, Canastar A, Dreskin SC. Anti-inflammatory effect of thymoquinone in a mouse model of allergic lung inflammation. Int Immunopharmacol 2006;6:1135-42.

12. Mansour MA, Tornhamre S. Thymoquinone inhibits 5lipoxygenase and leukotriene C4 synthase in human blood cell. J Enzym Inhib Med Chem 2004;19:431-6.

13. Al-Ghamdi M. Anti-inflammatory, analgesic and antipyretic activity of nigella sativa. J Ethnopharmacol 2001;76:45-8.

14. Odeh F, Ismail SI, Abu-Dahab R, Mahmoud IS, Al-Bawab A Thymoquinone in liposomes: a study of loading efficiency and biological activity towards breast cancer. Drug Delivery 2012;19:371-7.

15. Dhawan B, Aggarwal G, Harikumar S. Enhanced transdermal permeability of piroxicam through novel nanoemulgel formulation. Int J Pharm Investig 2014;4:65-76.

16. Shakeel $F$, Ramadan $\mathrm{W}$, Ahmed MA. Investigation of true nanoemulsions for the transdermal potential of indomethacin: characterization, rheological characteristics, and ex vivo skin permeation studies. J Drug Target 2009;7:435-41.

17. Shakeel F, Ramadan W, Rizwan M, Faiyazuddin M, Mustafa G, Shafiq S, et al. Transdermal and topical delivery of antiinflammatory agents using nanoemulsion/microemulsion: an updated review. Curr Nano Sci 2010;6:184-98.

18. Ahad A, Aqil M, Kohli K, Chaudhary H, Sultana Y, Mujeeb M, et al. Chemical penetration enhancers: a patent review. Expert Opin Ther Pat 2009;19:969-88.

19. Talegaonkar S, Mustafa G, Akhter S, Iqbal ZI. Design and development of oral oil-in-water nanoemulsion formulation bearing atorvastatin: in vitro assessment. J Dispersion Sci Technol 2009;30:1-12.

20. Liu Y, Yu XM, Sun RJ, Pan XL. Folate-functionalized lipid nanoemulsion to deliver chemo-radiotherapeutics together for 
the effective treatment of nasopharyngeal carcinoma. AAPS PharmSciTech 2017;18:1374-81.

21. Afzal SM, Shareef MZ, Dinesh T, Kishan V. Folate-PEG-decorated docetaxel lipid nanoemulsion for improved antitumor activity. Nanomedicine 2016;11:2171-84.

22. Constantinides PP. Lipid microemulsions for improving drug dissolution and oral absorption: physical and biopharmaceutical aspects. Pharm Res 1995;12:1561-72.

23. Lawrence MJ, Rees GD. Microemulsion-based media as novel drug delivery systems. Adv Drug Delivery Rev 2000;45:89:121.

24. Faiyazuddin M, Akhtar N, Akhter J, Shakeel F, Shafiq S, Mustafa $\mathrm{G}$, et al. Production, characterization, in vitro and ex vivo studies of babchi oil-encapsulated nanostructured solid lipid carriers produced by a hot aqueous titration method. Pharmazie 2010;65:347-54.

25. Ghosheh OA, Houdi AA, Crooks PA. High performance liquid chromatographic analysis of the pharmacologically active quinones and related compounds in the oil of the black seed (Nigella sativa L.). J Pharm Biomed Anal 1999;19:757-62.

26. Faiyazuddin M, Akhtar J, Mustafa G. Formulation of tea tree essential oil-loaded nanoemulsion system using aqueous titration method: In vitro and ex vivo kinetics. Int J Essential Oil Ther 2009;3:22-7.

27. Mustafa G, Khan ZI, Bansal T, Talegaonkar S. Preparation and characterization of oil in water nano-reservoir systems for improved oral delivery of atorvastatin. Curr Nano Sci 2009;5:428-40.

28. Mustafa G, Baboota S, Ahuja A, Ali J. Formulation development of chitosan-coated intra nasal ropinirole nanoemulsion for better management option of parkinson: an in vitro ex vivo evaluation. Curr Nano Sci 2012;8:348-60.

29. Mikolaj M, Yerramreddy TR, Ghosh P, Crooks PA, Stinchcomba AL In vitro permeation of a pegylated naltrexone prodrug across the microneedle-treated skin. J Controlled Release 2010;146:37-44.

30. Anwer MK, Jamil S, Ibnouf EO, Shakeel F. Enhanced antibacterial effects of clove essential oil by nanoemulsion. J Oleo Sci 2014;63:347-54.
31. Wintr CA, Risley EA, Nuss GW. Carrageenin-induced edema in hind paws of the rat as an assay for anti-inflammatory drugs. Proc Soc Exp Biol Med 1962;111:544-7.

32. Ghosh MN. Fundamentals of experimental Pharmacology. 3rd edition. Hilton and Company, Kolkata, India; 2005. p. 192-3.

33. Rao J, McClements DJ. Formation of flavor oil microemulsions, nanoemulsions and emulsions: influence of composition and preparation method. J Agric Food Chem 2011;59:5026-35.

34. Craig DQM, Barker SA, Banning D. An investigation into the mechanisms of self-emulsification using particle size analysis and low-frequency dielectric spectroscopy. Int J Pharm 1995;114:103-10.

35. Boucher EA. Separation of small-particle dispersions by the preferential accumulation in one of two liquid phases, or by static flotation at their interface. J Chem Soc Faraday Trans 1989;85:2963-72.

36. Bhardwaj A, Hartland S. Dynamics of emulsification and demulsification of water in crude oil emulsions. Ind Eng Chem Res 1994;33:1271-9.

37. Schleich N, Preat V. Nanostructures overcoming the skin barriers: Drug delivery strategy: In ch 6.2 by Alonso MJ, Csaba NS. Nanostructure biomaterials for overcoming biolologicas barriers: The Royal Society of Chemistry, UK; 2012.

38. Tan G, Xu P, Lawson LB, He J, Freytag LC, Clements JD, et al. Hydration effects on skin microstructure as probed by highresolution cryo-scanning electron microscopy and mechanistic implications to enhanced transcutaneous delivery of biomacromolecules. J Pharm Sci 2010;99:730-40.

39. Finlay TM, Abdulkhalek S, Gilmour A, Guzzo C, Jayanth P, Amith $\mathrm{SR}$, et al. Thymoquinone-induced Neu4 sialidase activates NFKB in macrophage cells and pro-inflammatory cytokines in vivo. Glycoconj J 2010;27:583-600.

40. Wang Y, Gao H, Zhang W, Zhang W, Fang L. Thymoquinone inhibits lipopolysaccharide-induced inflammatory mediators in BV2 microglial cells. Int Immunopharmacol 2015;26:169-73. 